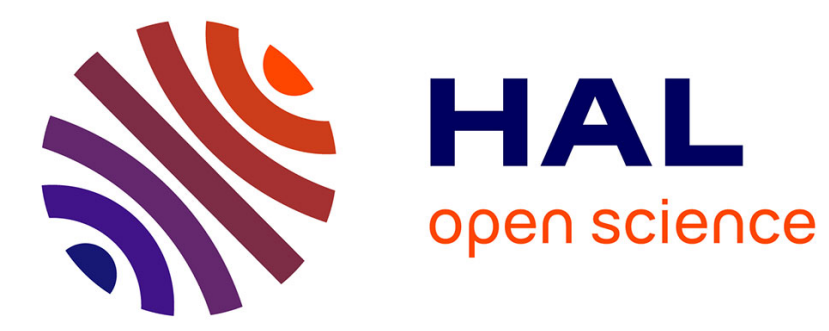

\title{
Relations entre dilatation et premiers stades de l'oxydation interne
}

\author{
L. Charrin, A. Combe, A. Charai, F. Cabane, J. Cabane
}

\section{To cite this version:}

L. Charrin, A. Combe, A. Charai, F. Cabane, J. Cabane. Relations entre dilatation et premiers stades de l'oxydation interne. Journal de Physique IV Proceedings, 1994, 04 (C3), pp.C3-41-C3-46. 10.1051/jp4:1994305 . jpa-00252502

\section{HAL Id: jpa-00252502 https://hal.science/jpa-00252502}

Submitted on 1 Jan 1994

HAL is a multi-disciplinary open access archive for the deposit and dissemination of scientific research documents, whether they are published or not. The documents may come from teaching and research institutions in France or abroad, or from public or private research centers.
L'archive ouverte pluridisciplinaire HAL, est destinée au dépôt et à la diffusion de documents scientifiques de niveau recherche, publiés ou non, émanant des établissements d'enseignement et de recherche français ou étrangers, des laboratoires publics ou privés. 


\title{
Relations entre dilatation et premiers stades de l'oxydation interne
}

\author{
L. CHARRIN, A. COMBE, A. CHARAI, F. CABANE et J. CABANE
}

Laboratoire de Métallurgie, EDIFIS, URA 443 du CNRS, Faculté des Sciences et Techniques de Saint Jérôme, case 511, 13397 Marseille cedex 20, France

\begin{abstract}
Variation of the lattice parameter during internal oxidation of dilute $\mathrm{Ag}(\mathrm{Mg})$ alloys is closely related to the successive steps of the oxidation process at low temperature. Models of elementary reactions are proposed by taking into account a potential barrier due to the strain field near the reacting species. First, substoichiometric species $([\mathrm{O}] /[\mathrm{Mg}]<1)$ form in a non expanded zone; then excess oxygen by comparison with the amount necessary to form stoichiometric $\mathrm{MgO}$ oxide and over stoichiometric clusters are responsible of the highly expanded zone observed. The decrease of deformation and of excess oxygen which occurs at last is related to an evolution toward more compact clusters.
\end{abstract}

\section{Introduction}

Lorsque l'oxygène pénètre dans un alliage, il peut produire une oxydation interne des éléments en solution plus oxydables que l'élément de base. Une couche où coexistent le métal de base et des particules d'oxyde, se développe à partir de la surface avec un front d'oxydation qui sépare la zone oxydée de la solution solide initiale (Fig.1).

La cinétique de l'oxydation interne a été étudiée théoriquement par Wagner /1/. Pour les alliages dilués, la théorie suppose la formation immédiate de l'oxyde stoechiométrique et par conséquent une cinétique d'oxydation gouvernée uniquement par la diffusion de l'oxygène. On obtient alors une loi en $t^{1 / 2}$ pour e l'épaisseur de la zone oxydée et pour $n_{\mathrm{O}} / \mathrm{S}$ le nombre d'atomes d'oxygène fixé par unité de surface:

$$
e=\left[\frac{2 C_{O} D_{O}}{v C_{M}}\right]^{1 / 2} t^{1 / 2} \quad \frac{n_{O}}{S}=\left[2 C_{O} D_{O} C_{M}\right]^{1 / 2}(v t)^{1 / 2}
$$

$C_{M}$ est la concentration de l'alliage en élément oxydable $M$,

$C_{O}$ est la solubilité de l'oxygène fixée par la pression d'oxygène et la température et $D_{O}$ le coefficient de diffusion de l'oxygène,

$v=[O] /[M]$ définit la stoechiométrie de l'oxyde formé $\mathrm{MO}_{v}$.

Cette théorie est bien vérifiée lorsque l'oxydation a lieu à haute température. 


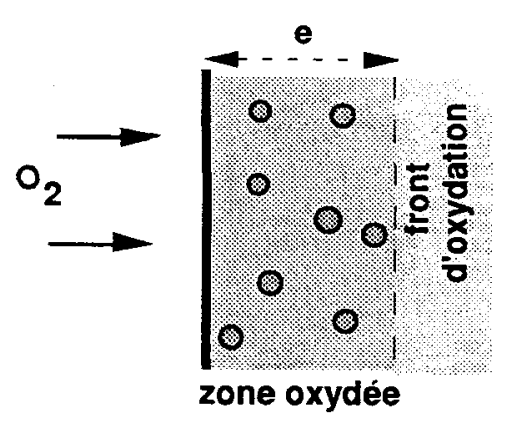

Figure 1 - Représentation schématique de l'oxydation interne à haute température.

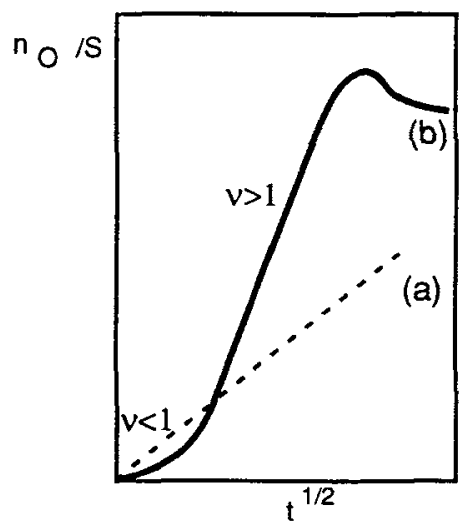

Figure 2 - Cinétique de fixation de l'oxygène à basse température:

(a) courbe théorique pour l'oxyde $\mathrm{MgO}$;

(b) courbe expérimentale correspondante.

Aux basses températures, on pourrait s'attendre à avoir une cinétique de fixation de l'oxygène ralentie par la réaction d'oxydation. En fait la fixation de l'oxygène est immédiate comme le prévoit la théorie de Wagner, mais la variation en fonction du temps de la quantité d'oxygène fixé $n_{\delta} / S$ ne suit pas la loi de Wagner $/ 2 /, / 3 /, 14 /$. Les écarts observés sont attribués à une variation du rapport $[\mathrm{O}] /[\mathrm{M}]=v$ en cours d'oxydation (Fig. 2). En même temps, la résistivité résiduelle, la dureté et le paramètre de la solution solide augmentent, passent par un maximum et se fixent définitivement à des valeurs très élevées incompatibles avec des précipités d'oxyde inclus dans un métal pur $/ 4 /, / 5 /, / 6 /, / 7 /$. De plus, des images obtenues en microscopie électronique en transmission montrent que les particules formées ont une taille inférieure à $1 \mathrm{~nm} / 8 /$. Ces résultats ont été obtenus pour différents systèmes; ils ont conduits aux conclusions suivantes /2/,19/:

- aux basses températures, l'état stable avec formation de la phase constituée par des précipités de l'oxyde stoechiométrique, n'est pas atteint; l'état final correspond à un état métastable;

- les produits de l'oxydation sont des espèces en solution qui résultent d'associations entre des atomes métalliques en substitution et des atomes d'oxygène en insertion;

-la stoechiométrie de ces espèces varie avec le degré d'évolution du système.

Différents stades de l'oxydation interne aux basses températures ont été identifiés à l'aide des résultats obtenus avec des techniques complémentaires, macroscopiques et microscopiques: gravimétrie, calorimétrie, résistivité, microscopie électronique en transmission./2/,/3/,/4/ Ces stades sont visibles sur la figure 2 qui montre la cinétique de fixation d'oxygène observée dans un alliage $\mathrm{Ag}(\mathrm{Mg})$ et la courbe prévue par la théorie de Wagner avec formation de l'oxyde $\mathrm{MgO}$ :

1er stade: formation d'espèces oxydées sous stoechiométriques $(v<1)$, associée à une cinétique est plus lente que la cinétique théorique calculée pour $v=1$;

2ème stade: évolution vers des amas avec un excès d'oxygène $(v>1)$, seule explication possible pour une cinétique plus rapide que celle prévue par la théorie de Wagner;

3ème stade: ré-arrangements et relaxation des amas avec élimination partielle de l'excès d'oxygène, état final aux basses températures. 
Ces phénomènes se produisent également à haute température $/ 7 /$ mais l'évolution se poursuit ensuite vers la formation de l'oxyde massif stoechiométrique.

Dans cette communication, nous portons notre attention sur les phénomènes de dilatation qui se produisent pendant l'oxydation afin d'obtenir des précisions sur la nature des espèces formées aux basses températures. Notre travail porte sur la solution solide $\mathrm{Ag}(\mathrm{Mg})$ $(0,5$ et 1 at. $\%)$ oxydée entre 280 et $420^{\circ} \mathrm{C}$.

\section{Mesure du paramètre cristallin de la solution solide.}

Les déterminations de paramètre cristallin sont faites sur des plaquettes polycristallines par diffraction des rayons $X$. Nous avons étudié les variations de paramètre à la surface des échantillons en fonction du temps d'oxydation; nous avons également mesuré le paramètre en fonction de la distance à la surface en faisant des diagrammes après dissolution progressive d'échantillons partiellement oxydés /10/.

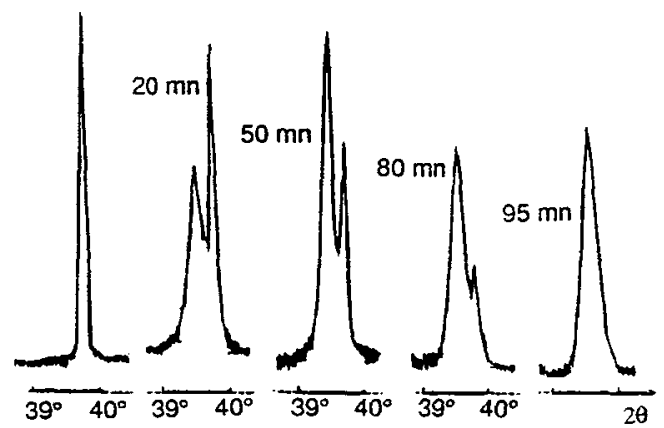

Figure 3 - Diffraction des rayons $X$ : évolution de la raie $(200)$ à la surface d'un échantillon.

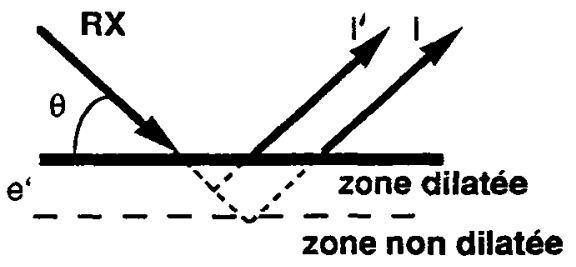

Figure 4 - Schéma du montage de diffraction utilisé pour déterminer les paramètres cristallins

La figure 3 montre la raie (200) de la solution solide avant oxydation et l'évolution du diagramme après différents temps d'oxydation à température et pression d'oxygène constantes. On constate l'apparition d'une raie supplémentaire et au bout d'un certain temps seule cette raie supplémentaire subsiste; elle correspond à une très forte dilatation du réseau. Les mêmes observations peuvent être faites sur les autres raies de diffraction.

Avec le montage utilisé (Fig. 4), une variation continue de paramètre avec la profondeur se traduirait par un déplacement progressif de la raie (200) accompagné d'un élargissement. Le dédoublement des raies (Fig.3) montre qu'il existe une limite nette ou front de dilatation entre une zone dilatée proche de la surface et une zone non dilatée située en dessous. Lorsque la zone dilatée est inférieure à quelques microns, les pics de diffraction provenant des deux zones sont observables simultanément; il est possible de mesurer l'épaisseur e' de la zone dilatée d'après les intensités des raies correspondant respectivement à la zone dilatée et à la zone non dilatée, en tenant compte de l'angle de diffraction et du coefficient d'absorption pour le rayonnement utilisé. Lorsque la zone dilatée devient plus épaisse, la raie de diffraction de la partie non dilatée devient invisible aux rayons $X$.

\section{Résultats}

Les paramètres cristallins obtenus en surface après oxydation à $300^{\circ} \mathrm{C}$ sous une 
pression d'oxygène de 0,2 atm.,d'un alliage à 1 atome\% de magnésium sont représentés en fonction du temps d'oxydation sur la figure 5; ceux obtenus après dissolution sont portés en fonction de l'épaisseur enlevée sur la figure 6. Il faut d'abord remarquer que les premiers effets de l'oxydation sur les paramètres cristallins doivent être recherchés au début des courbes de la figure 5 pour des temps courts, alors qu'ils apparaissent à la fin des courbes de la figure 6 , lorsque la solution solide est visible aux rayons $X$. Ces variations de paramètres montrent donc que:

- la dilatation n'apparaît pas au début de l'oxydation (fig. 5);

- quand la dilatation apparaît, le paramètre augmente rapidement, passe par un maximum, décroît et prend une valeur constante (Fig. 5 et 6);

- l'état final est caractérisé par une forte dilatation par rapport à la solution solide initiale.

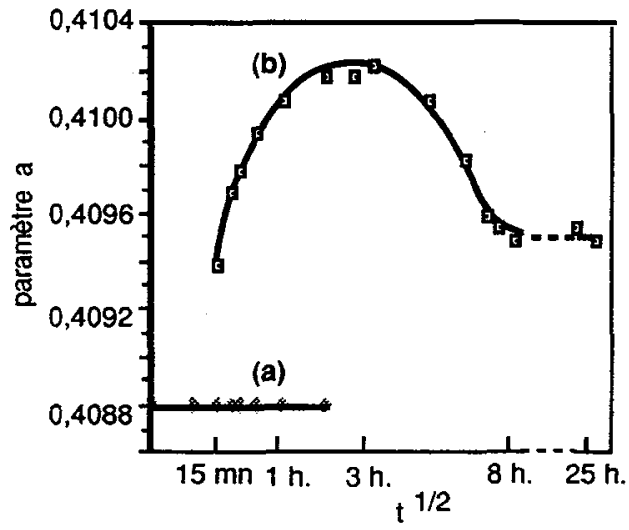

Figure 5 - Paramètre cristallin mesuré en surface après différents temps d'oxydation. $\mathrm{Ag}-\mathrm{Mg}(1 \mathrm{at} . \%), 300^{\circ} \mathrm{C}, \mathrm{pO}_{2}=0,2 \mathrm{~atm}$.

a) couche non dilatée (solution solide),

b) couche dilatée.

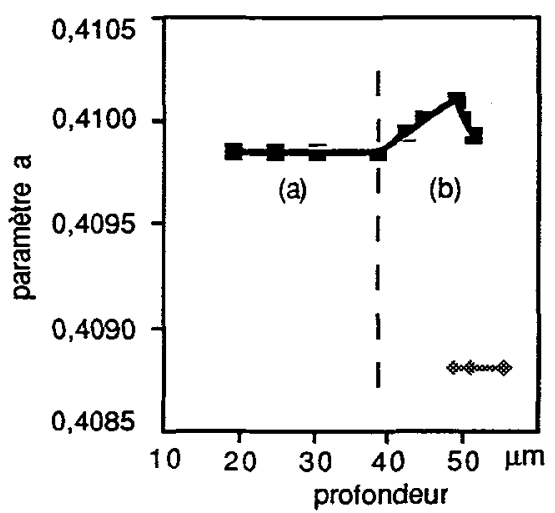

Figure 6 - Paramètre cristallin en fonction de la profondeur après $92 \mathrm{~h}$. d'oxydation : (a) zone oxydée à l'état métastable; (b) zone en évolution

\section{Discussion et interprétation}

Sur la figure 7, nous comparons la quantité d'oxygène fixé et le nombre d'atomes de magnésium contenus dans la zone dilatée calculé d'après l'épaisseur de cette zone. Nous constatons que la dilatation n'est pas perceptible avant $15 \mathrm{mn}$ d'oxydation alors que des quantités importantes d'oxygène ont déjà été fixées. Elle apparaît lorsque la cinétique de fixation de l'oxygène (Fig. 2) s'accélère avec la formation d'amas contenant un excès d'oxygène. Nous en déduisons que les espèces sous-stoechiométriques initiales déforment peu le réseau cristallin de la solution solide.

Le front d'oxydation apparaît alors comme une zone diffuse qui contient des espèces sous-stoechiométriques sans dilatation appréciable du réseau. Plus près de la surface, il y a une zone dilatée avec des amas sur-stoechiométriques (Fig. 8). 


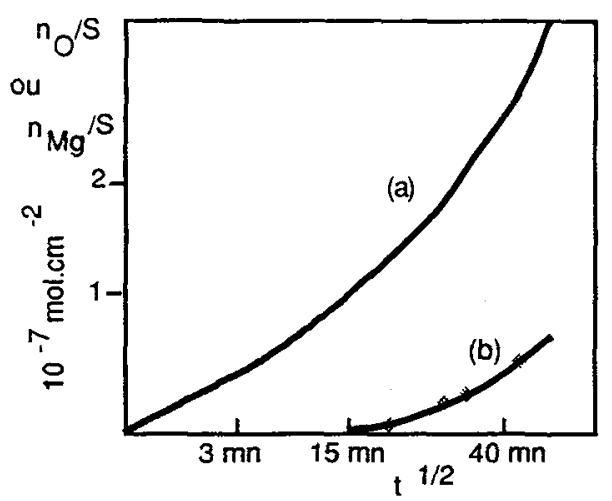

Figure 7 - Comparaison entre la quantité d'oxygène fixé (a) et la quantité de magnésium dans la zone dilatée (b). $\mathrm{Ag}(\mathrm{Mg}) 0,5$ at. $\%, 280^{\circ} \mathrm{C}, \mathrm{pO}_{2}=1$ atm.

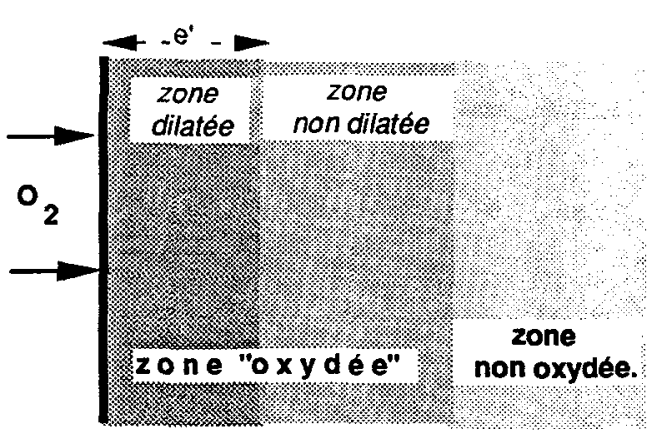

Figure 8 - Oxydation interne à basse température: schéma de la zone oxydée.

Tous les résultats relatifs aux variations du rapport $\mathrm{no}_{\mathrm{O}} / \mathrm{n}_{\mathrm{M}}$ en fonction des différents paramètres $\left(t, \mathrm{C}_{\mathrm{M}}, \mathrm{PO}_{2}, T\right)$ montrent qu'il existe un rapport étroit entre la dilatation et l'excès d'oxygène. En revanche les espèces sous-stoechiométriques déforment peu le réseau cristallin. Cela s'explique bien si on tient compte de la forte déformation due à l'oxygène en insertion et de la liaison $\mathrm{Mg}-\mathrm{O}$ beaucoup plus forte donc plus courte que la liaison $\mathrm{Ag}-\mathrm{O}$. En conséquence, la déformation diminue chaque fois qu'un atome d'argent est remplacé par un atome de magnésium, près d'un atome d'oxygène. Ainsi, il est clair que la déformation est moins importante pour un amas terminé par O-Mg (Fig. 9 a) que pour un amas terminé par Mg-O (Fig. 9b). Il y a donc une corrélation étroite entre faible dilatation et nombre élevé d'atomes de magnésium à la périphérie des amas d'une part et entre forte dilatation et nombre élevé d'atomes d'oxygène à la périphérie des amas d'autre part. La première situation correspond à des amas sous-stoechiométriques, la seconde à des amas sur-stoechiométriques.

Avant de proposer un modèle de réactions élémentaires, rappelons que la fixation d'oxygène est immédiate (calorimétrie et gravimétrie). Ceci nous autorise à négliger les réactions inverses des premières réactions élémentaires et à prendre comme étape initiale, la formation de l'espèce $\mathrm{MgO}$ obtenue par fixation irréversible d'un atome d'oxygène près d'un atome de magnésium. Parmi les réactions suivantes, on a le choix entre la fixation d'atomes d'oxygène ou d'atomes de magnésium sur cette première espèce (Fig. 10).

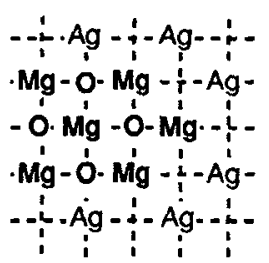

(a)

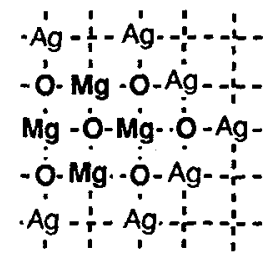

(b)

Figure 9 - Schémas représentant les

amas: a) sous- stoechiométriques;

b) sur-stoechiométriques.

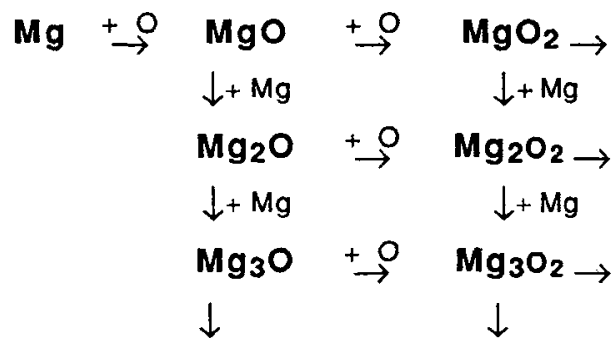

Figure 10- Schéma des réactions élémentaires. 
La cinétique d'une réaction dépend de la probabilité de rencontre entre deux espèces, définie par leurs concentrations et leurs mobilités, et de la barrière de potentiel qui doit être franchie lors de l'établissement de la nouvelle liaison. Les déformations et les champs de contrainte associés jouent donc un rôle essentiel pour orienter la suite des réactions. Si on compare, par exemple, la réaction $\mathrm{MgO}+\mathrm{O}$ et la réaction $\mathrm{MgO}+\mathrm{Mg}$, on doit s'attendre à une barrière de potentiel due au champ de contrainte beaucoup plus faible dans le second cas que dans le premier parce que le magnésium déforme beaucoup moins le réseau que l'oxygène. De plus, il n'est pas exclu que la formation de l'espèce $\mathrm{MgO}_{2}$ ne soit pas possible du fait de la haute densité de contrainte liée à sa présence dans le réseau. Ainsi la fixation d'atomes de magnésium est privilégiée et les premières réactions élémentaires conduisent à la formation d'espèces $\mathrm{Mgm}_{\mathrm{m}} \mathrm{O}_{\mathrm{n}}$ sous-stoechiométriques ( $\mathrm{n}$ et $\mathrm{m}$ sont des valeurs moyennes). Tant quil existe du magnésium libre, le premier stade se déroule avec la formation de ces espèces.

Quand il n'y a plus d'espèces mobiles autres que l'oxygène, celui-ci peut se fixer, $\mathrm{m}$ reste constant alors que $n$ augmente tant que la déformation reste supportable par le réseau cristallin. L'oxygène en excès se place à la périphérie de ces amas.

Le dernier stade observé est un stade de relaxation beaucoup plus lent avec un dégagement de chaleur notable, témoin d'une augmentation du nombre des liaisons $\mathrm{Mg}-\mathrm{O}$ et une diminution de l'oxygène en excès; il s'explique par une diminution du rapport surface/volume et un accroissement de la compacité des amas.

En conclusion, l'étude des variations du paramètre cristallin de la solution solide $\mathrm{Ag}(\mathrm{Mg})$ au cours de loxydation interne aux basses températures a mis en évidence l'existence d'un front de dilatation et d'une zone non dilatée dans laquelle apparaissent les premières manifestations de la réaction d'oxydation sous la forme d'espèces sous- stoechiométriques. En associant ces résultats à ceux précédemment obtenus à l'aide d'autres techniques, nous avons proposé un schéma de réactions élémentaires en solution qui explique la formation initiale de ces espèces en prenant en compte le rôle important du champ de déformation lié à la présence de l'oxygène en insertion. Ainsi en étudiant loxydation interne dans le domaine des basses températures, nous avons suivi une transformation de phase à l'échelle atomique qui préfigure la formation d'une interface oxyde-métal.

\section{REFERENCES}

1 - C. WAGNER, Z. Elektrochem., 63, 772, (1959)

2 - L. ChARRIN, A. COMBE et J. CABANE, Oxid. Met., 37, 65, (1992)

3 - B. M. SEMEGA, L. CHARRIN, A. COMBE et J. ARIDE, Phil. Mag. A, 66, 1139, (1992)

4 - L. ChARRIN, A. COMBE, A. ChARAI et J. CABANE, (ce colloque)

5 - J. L. MEIJERING et M. J. DRUYVESTEYN , Philips. Res. Rep., 2, 81, (1947)

6 - M. J. KLEIN et R. A. HUGGINS, Acta Metall., 10, 55, (1962)

7 - A. COMBE et J. CABANE, Oxid. Met., 21, 21, (1984)

8 - A. Charal et G. NIHOUL, Phil. Mag. A, 58, 571, (1988)

9 - L. S. DARKEN, Trans. Am. Soc. Met., 54, 600, (1961)

10 - L. CharRin, A. COMBE, F. CABANE et J. CABANE, Oxid. Met., (sous presse) 